Al final del pasillo. Modalidades de lo siniestro en el videojuego

Diego Maté

Boletín de Arte (N. $\left.{ }^{\circ} 19\right)$, e015, septiembre 2019. ISSN 2314-2502

https://doi.org/10.24215/23142502e015

http://papelcosido.fba.unlp.edu.ar/ojs/index.php/boa

Facultad de Bellas Artes. Universidad Nacional de La Plata

La Plata. Buenos Aires. Argentina

\title{
AL FINAL DEL PASILLO MODALIDADES DE LO SINIESTRO EN EL VIDEOJUEGO
}

\section{AT THE END OF THE HALLWAY \\ MODALITIES OF THE UNCANNY IN VIDEO GAMES}

\author{
Diego Maté \\ diegomateyo@gmail.com
}

Instituto de Investigación y Experimentación en Artes y Crítica. Universidad Nacional de las Artes. Argentina

Recibido: 21/12/2018

Aceptado: 16/3/2019

\section{RESUMEN}

En la actualidad, la expansión de la producción independiente y la apropiación de operaciones provenientes de los lenguajes artísticos le permiten al videojuego experimentar con nuevas formas lúdicas. Dentro de la escena del walking simulator, cada vez más juegos actualizan la categoría de lo siniestro. En los casos analizados se parte de un motivo común, el de la casa, para indagar en distintas formas de enrarecimiento del espacio, del relato, de los temas y de la enunciación. El tratamiento de esas dimensiones instala una incertidumbre entre la existencia de amenazas y peligros que emergen de algo conocido, que resulta familiar, y cuya naturaleza oscila entre lo real y lo imaginario.

Palabras ClaVe

Siniestro; videojuego; arte; fantástico; ort gome

\section{ABSTRACT}

At present, the growth of independent production and the appropriation of operations coming from artistic languages allow the video game to experiment with new ludic forms. Within the walking simulator scene, more and more games update the category of the uncanny. In the cases analyzed, the starting point is a common motif: that of the house, to investigate different forms of rarification of the space, the story, the themes and the enunciation. The treatment of these dimensions introduces an uncertainty between the very existence of threats and dangers that emerge from something known and familiar, and whose nature oscillates between the real and the imaginary.

\section{KEYWORDS}

Uncanny; videogame; art; fantastic; art game 
En los últimos años, el videojuego ensayó nuevas aproximaciones a lo siniestro. Si bien pueden rastrearse casos en el pasado, fue solo recientemente que el medio empezó a experimentar de manera sostenida las potencialidades de esa categoría estética. Estos primeros esbozos están en diálogo con una serie de transformaciones también recientes y son su resultado. Primero, el surgimiento de una zona de producción independiente, distanciada de los mandatos de las producciones $\mathrm{AAA}^{1}$ de los grandes estudios de desarrollo: la consolidación del sector indie supuso un notorio incremento de la posibilidad de experimentación con formas lúdicas nuevas (Donovan, 2010). Segundo, a partir del nuevo milenio, se generan intercambios crecientes entre el campo de las artes y el videojuego. Estos cruces adoptan configuraciones múltiples, pero a grandes rasgos pueden detectarse tres regímenes de intercambio recurrentes: la exhibición de juegos en museos y galerías; la apropiación de elementos del videojuego por parte de artistas; y la aparición del art gome, una clasificación poco definida pero de gran eficacia que rotula juegos y los separa del resto de la producción mediática (Maté, 2019). Tercero, dentro del terreno del art game, la estabilización de una forma lúdica inédita llamada walking simulator² se volvió la condición de posibilidad de una buena parte de las investigaciones estéticas y lúdicas más interesantes (Cross, 2015), dentro de las cuales se inscriben las reformulaciones de lo siniestro que analizaré en este trabajo.

Lo siniestro ha sido problematizado largamente, por lo que no profundizaré en la cuestión. Del trabajo seminal de sigmund Freud sobre lo siniestro en 1919, en el que el autor vuelve sobre planteos de Ernst Jentsch, la noción ha sido objeto de rescates y relecturas de todo tipo. Para Jan Masschelein (2011), el concepto cobra una centralidad definitiva en la cultura recién en los años setenta. Las perspectivas deconstructivistas lo llevaron hacia el terreno de los textos marginales; otros abordajes lo buscaron en el terreno de la literatura fantástica y en formas de alienación denunciadas por el marxismo. En los noventa, lo siniestro se estabiliza y expande desde la teoría crítica y los estudios literarios a los dominios del arte, donde pasa a organizar tanto la producción como el reconocimiento. La autora explica que la noción se vuelve lo suficientemente plástica como para caracterizar fenómenos políticos, históricos, humanísticos, tecnológicos, psíquicos, religiosos, etcétera. Su utilización exhibe oscilaciones conceptuales, pero el significante, pegajoso, permanece y se ramifica. Las derivas de lo siniestro alcanzan a los estudios de género y poscoloniales, donde la mujer y el extranjero son interpretados como lo reprimido que vuelve y resulta inquietante (Masschelein, 2011).

Anthony Vidler (1992) entiende lo siniestro como signo de una condición moderna cuyo núcleo reside en la experiencia de una burguesía en ascenso que sufre, en términos psíquicos, el contraste entre la seguridad interior provista por la propia casa y el miedo a amenazas que provienen del exterior. Vidler explica que el concepto puede ser leído como síntoma de que el hombre nunca puede hacer plenamente del mundo su hogar. Según el autor, ese extrañamiento del mundo tuvo su reflejo epocal en las vanguardias históricas y en sus estrategias de enrarecimiento y desnaturalización.

A lo largo de este trabajo, retendré la definición freudiana de lo siniestro como efecto que se produce cuando lo familiar se vuelve extraño (Freud, 1992). Si bien Freud problematiza aspectos psicoanalíticos, lo hace analizando cuentos y novelas, es decir, que le reconoce al fenómeno una vida textual y procedimientos formales (como la oscilación entre la representación de un mundo real y otro fantástico). Desde una perspectiva sociosemiótica, lo siniestro será entendido como un efecto sostenido en una multiplicidad de operaciones discursivas localizables en diferentes niveles de los textos. Dejaré de lado las elaboraciones psicoanalíticas del concepto para centrarme en la descripción de rasgos con el fin de comprender la

1 Juegos de gran presupuesto, por lo general realizados por equipos de cientos de personas.

2 se trata de una clasificación que rubrica juegos donde se atenúan (o desaparecen) las estructuras lúdicas y se privilegian elementos exploratorios. La etiqueta surgió con un tinte despectivo, pero con el incremento de la producción de walking simulators pasó a funcionar de manera descriptiva. 
generación de escenas enunciativas caracterizadas por la ambivalencia y la incertidumbre. ${ }^{3}$ Se trata de pactos comunicativos propuestos solo recientemente por el videojuego y que surgen de articulaciones específicas de operaciones relacionadas con el espacio, el relato, la interacción y lo lúdico. El conjunto de esas operaciones introduce desvíos respecto de la producción mainstream y de los géneros populares del medio: como se verá, es en buena medida ese desvío lo que posibilita la emergencia de lo siniestro. ${ }^{4}$

\section{GONE HOME}

En Gone Home (GH) (2013), del estudio Fullbright, un walking simulator de gran popularidad, se anuncia lo que vendrá tiempo después. El juego cuenta la historia de Katie Greenbriar, que después de estudiar un año en el exterior vuelve con su familia. En ese lapso los Greenbriar se mudaron, por lo que Katie arriba a una casa nueva y, para ella, desconocida. A su llegada no hay nadie para recibirla y la protagonista debe entrar en la casa por su cuenta y reconstruir todo un año de la vida familiar mediante la observación del entorno y de los objetos, la lectura de cartas, notas y cuadernos, etcétera. A medida que avanza la pesquisa por la casa se comprende que se está ante una propuesta singular: el recorrido puede hacerse en cualquier orden, no hay prácticamente obstáculos y, ciertamente, no se verifican peligros; por lo tanto, no se puede ganar ni perder. Se trata de un terreno discursivo nuevo para el videojuego: $G H$ se aleja de los contratos lúdicos de los géneros tradicionales que caracterizaron la historia del medio desde la segunda mitad de los setenta; las convenciones fuertes caen y se propone, en su lugar, una experiencia exploratoria y no lúdica (Maté, 2017).

El universo de ese relato a reconstruir supone también una novedad para el medio: se trata de un drama adolescente situado en los noventa y atravesado por conflictos familiares y amorosos en un contexto socioeconómico de clase media que son trabajados de manera naturalista (Sloan, 2014). Sin embargo, a pesar de que el espacio de la casa no alberga peligro alguno, el recorrido produce un efecto frecuente: Ios pasillos largos, las habitaciones abiertas, los dispositivos electrónicos que quedaron encendidos (un televisor, una radio), las luces, todo puede volverse fuente de una amenaza no identificada. El tratamiento naturalista, lejos de obstaculizar la generación del efecto, lo habilita: es la experiencia misma del espacio, del desplazamiento por el entorno de una casa prototípica, lo que produce esa sensación de acechanza. El recorrido instaura esa tensión y no la resuelve: la impresión de que algo persigue, la inminencia de algo terrible nunca es confirmada, pero el efecto no se disipa, su potencia radica en que, sin importar la situación diegética, el efecto persiste.

La puesta en obra de lo siniestro de GH surge del trabajo con recursos específicos del videojuego. La tematización de lo cotidiano no explica por sí sola el extrañamiento, es necesario dar cuenta, además, de una forma de contacto con el entorno y, a su vez, de una historia textual activada por la representación. En este sentido, puede tomarse para el videojuego lo sostenido por Carolina Bejarano (2011) para el cine: la autora afirma que lo siniestro fílmico puede funcionar más allá de los motivos analizados por el psicoanálisis (como el doble ${ }^{67}$ la pérdida de la visión), atendiendo al despliegue de recursos específicamente cinematográficos. También en el videojuego, el efecto no preexiste a la representación, sino que emerge de ella y de su trabajo. 0, con otras palabras: «No es simplemente la experiencia la que nos

3 En discusión con la utilización psicoanalítica del concepto, Carolina Bejarano (2011) explica que se puede asumir el partido opuesto al de esa tradición: «Es posible hacer el camino inverso. Es decir, no tomar elementos de estos discursos para pensar psicoanalíticamente, sino recurrir a elementos y conceptos psicoanalíticos para dar cuenta de cómo se producen determinadas experiencias estéticas» (s. p.).

4 Ha habido trabajos, como el de Ashley Brown y Björn Marklund (2015), que analizaron lo siniestro como emergente imprevisto en el marco de juegos dirigidos a un público infantil como Animal Crossing. Allí, el efecto no es la resultante de un plan textual (como los casos que trabajaré), sino de agrietamientos casuales, no controlados, de dimensiones que hacen al contacto, por ejemplo, con los NPC (personajes no jugables). 
recuerda lo siniestro, sino la representación o la ficcionalidad, la retórica de que se nos recuerde la distancia entre la realidad y la ficción» (Aardse, 2014, p. 5).8

\section{All the Delicate Duplicates}

En el caso de GH, lo siniestro surge de manera dispersa y secundaria, como un efecto residual de la pesquisa. En All the Delicate Duplicates (AtDD) (2017), en cambio, lo siniestro adquiere un carácter global que se sustenta en una multiplicidad de operaciones. El juego de Mez Breeze y Andy Campbell posee, en principio, una estructura similar a la de GH: se debe recorrer un entorno en busca de pistas que permitan acceder a un relato ausente. En este sentido, los dos juegos presentan rasgos similares a lo que Tzvetan Todorov (1992) Ilama policial de misterio, variante del género en el que un relato, el de la investigación, permite develar y recomponer otro, el del crimen. Sin embargo, en los casos analizados no hay en verdad dos relatos, sino uno, el que debe reconstruirse, ya que las pesquisas en sí mismas no están organizadas narrativamente, sino que proponen una experiencia exploratoria (Maté, 2017).

En AtDD, John es ingeniero informático y vive con su hija Charlotte, que estudia artes. Un día a John se le informa que heredará una pequeña colección de objetos de la tía Mo, una pariente lejana que el protagonista no recuerda. Los objetos poseen un aura extraña que fascina a Charlotte: la hija se obsesiona con ellos y los utiliza como modelos para sus clases. La presencia de esos objetos en la casa y en la vida de los personajes produce un enrarecimiento que crece con los años. El departamento se vuelve el espacio de escenas imposibles a las que John asiste incrédulo; las habitaciones son invadidas subrepticiamente por entes y masas orgánicas indescriptibles. Los objetos podrían estar funcionando como catalizadores de alguna fuerza innombrable, como un puente a otro plano de existencia que se solapa con el de los protagonistas y lo corroe de a poco; o todo podría reducirse a un problema psiquiátrico de John.

En principio, AtDD pareciera trabajar de manera explícita sobre la noción de Freud de lo siniestro, en tanto el juego retrata un espacio cotidiano que se enrarece a partir del ingreso en el hogar de una serie de objetos misteriosos. Pero esta tematización de lo cotidiano enrarecido sirve de soporte a tres operaciones textuales: los cambios temporales bruscos, la descomposición en aumento del espacio del hogar y la confusión que emerge del relato reconstruido. Estas operaciones, sumadas a la tematización explícita del extrañamiento de lo cotidiano, elaboran lo siniestro en torno a una incertidumbre: la progresiva contaminación de lo real, ¿sucede efectivamente, o es el resultado de la psiquis precaria de John? El departamento se muestra cada vez más tomado por toda clase de elementos perturbadores cuya naturaleza se ignora, pero esto no disipa la duda; al contrario, es esa incertidumbre la que vehiculiza la inquietud. En este sentido, AtDD se inscribe con claridad en el registro de lo fantástico, género que, según Todorov (2011), se caracteriza por la vacilación respecto de los acontecimientos ocurridos en el relato. Lo fantástico ofrece un mundo ficcional cuyo orden tambalea ante la irrupción de hechos insólitos: mientras la incertidumbre en relación con lo sucedido y con sus efectos no se esclarece, se está en el terreno inestable de lo fantástico, aunque una explicación narrativa, sostiene Todorov, puede hacer que la obra se vuelque hacia lo maravilloso o, por el contrario, hacia lo extraño.

La de AtDD es, en todo caso, una apropiación no narrativa de lo fantástico, es decir, una transpolación del género a una discursividad predominantemente espacial, donde lo siniestro surge ya no del relato, sino de la experiencia del recorrido y del examen de un entorno explorable. Este pasaje mediático, aún con esos cambios, conserva aspectos del género en el nivel de los temas y de la enunciación. Todorov explica que, en lo fantástico, la vacilación de los personajes se vuelve una materia semántica trabajada por la obra. En AtDD, esa vacilación es modelada mediante el tema del resquebrajamiento de la percepción, en el que un deterioro del protagonista, ya sea psíquico o anímico, no permite diferenciar entre lo real y la alucinación. Esa incertidumbre, dice Todorov, se traslada del protagonista al lector, que como aquel debe elegir, 
optar por una explicación posible, aunque sea momentáneamente: lo sucedido se reduce a un problema perceptual, o bien tiene una lógica que escapa a la del mundo conocido. La ambivalencia deviene rasgo enunciativo que caracteriza la escena comunicacional desplegada por el género. AtDD sostiene ese contrato propio del género fantástico al no proveer ninguna explicación concluyente respecto de lo que sucede en el departamento y el enunciatario habita esa incertidumbre insoluble.

Si en GH lo siniestro emergía de manera lateral como resultado de una memoria textual, en AtDD, en cambio, lo siniestro, por la vía de lo fantástico, modela tanto el tema como la enunciación, es decir, gana la escena y deviene un efecto global que organiza la superficie discursiva en su conjunto.

\section{ANATOMY}

Hasta aquí pudo verse cómo la elaboración de lo siniestro partía de diferentes estrategias textuales que manipulaban de una u otra forma el espacio de una casa. En Anatomy (2016), de la desarrolladora independiente Kitty Horrorshow, todo comienza con el sonido de un casete siendo introducido en una videocasetera: la imagen deja ver un entorno tridimensional, explorable, velado por el filtro de la imagen de un VHS dañado. La casa está casi a oscuras y, para orientarse, hay que moverse pegado a las paredes. El juego no ofrece nada parecido a un objetivo a cumplir, por lo que los recorridos a lo largo de la casa, como en los casos anteriores, son libres y no existe el riesgo de perder.

Se llega a la cocina y allí se encuentra un reproductor y un casete de audio. Una vez introducido el casete, del aparato se escucha una voz femenina que habla de las casas, de su significación antropológica, de su fuerza simbólica. El audio termina y el juego comunica una tarea: «Hay un casete en el comedor». Una vez encontrado y escuchado el segundo casete, la secuencia se repite. Cada nuevo audio resulta más inquietante que el anterior, y tanto la casa como la imagen se degradan progresivamente. Esto ocurre varias veces hasta que en una excursión al sótano, el juego se cierra abruptamente. Pero ese no es el final. Una vez que se accede de nuevo al juego, se retoma la partida y todo parece haberse deteriorado aún más: el desgaste de la imagen dificulta la percepción del entorno; los audios solo dejan escuchar frituras y ruidos; finalmente, la casa misma se deshace, como si fuera consumida por glitchs, errores de programación que muestran un espacio en descomposición.

Si bien los casos anteriores no eran predominantemente narrativos, ofrecían historias cuyos trazos diseminados por el espacio permitían recomponer un relato ausente. En Anatomy, en cambio, no queda resto alguno de narratividad: solo un ovator que se mueve por una casa (de la que no se sabe ni siquiera si es suya ni por qué está ahí). La casa parece lo suficientemente prototípica: tiene dos plantas, un living, una cocina, tres habitaciones, dos baños, un sótano. Pero la presentación del lugar transmuta ese aire típico en un conjunto amenazante.

El mecanismo del juego puede leerse como la puesta en discurso de una concepción tradicional de lo siniestro, la que sostiene que el efecto surge del extrañamiento del hogar, espacio de intimidad y seguridad que se transforma, de un momento a otro, en una fuente de angustia. Vidler (1992) explica que esa inestabilidad de lo que parece familiar, íntimo y que se vuelve contra sus dueños hace tambalear la naciente institución del hogar burgués en el siglo XIX; el efecto se transforma así en síntoma de un malestar propio de la modernidad, producto de los miedos de una clase social que no termina todavía de afianzarse. El hogar, que debía proteger a sus miembros de los peligros de lo real, aislarlos del exterior, deviene una fuente nueva de angustia.

Anatomy representa esa escena primordial: a falta de datos narrativos elementales, solo quedan la casa y su habitante, que no es personaje (porque no se sabe nada de él o de ella), 
sino más bien un insumo textual que posibilita el recorrido. La casa no posee nada fuera de lo común, pero la oscuridad circundante obtura cualquier posible impresión de familiaridad o de seguridad. La situación se completa con la diseminación de los casetes y con los audios grabados, que dan cuenta de un asedio silencioso cuya fuente es inhallable. No se sabe cómo llegan los casetes a las habitaciones ni de quién es la voz y en los recorridos no se encuentran peligros de ningún tipo.

Una multiplicidad de operaciones sustentan lo siniestro en Anatomy: la casa se transforma en un territorio amenazado por obra de la soledad y de la oscuridad pero, especialmente, debido a la repetición. La secuencia de diseminación, búsqueda y escucha de los casetes impone la reiteración como principio organizador de la experiencia. Ya Freud señalaba que la repetición poseía un carácter siniestro porque evoca en la vigilia la sensación de inermidad propia de los estados oníricos (Freud, 1992). ${ }^{9}$

Pero la casa, a su vez, es un disparador, un insumo que vehiculiza el trabajo expresivo con distintos soportes mediáticos: los casetes de audio y la imagen de VHS permiten experimentar con un extrañamiento de tipo tecnológico, una forma particular de lo siniestro que para Kriss Ravetto-Biagioli (2016) surge en el arte contemporáneo mediante el trabajo con nuevos dispositivos y soportes. Así es que se ignoran las causas de la descomposición general, tanto de los audios, de la imagen y de la casa misma; eso no hace más que acrecentar la impresión de un peligro difuso, un clima propio del glitch horror, tendencia en auge que explota los miedos que surgen en torno de la falibilidad de la tecnología (Crawford, 2017). De esta forma, en Anatomy lo siniestro adquiere una escala nueva: el miedo surge de la inminencia de un derrumbamiento de materia textual, ya que son los soportes representados (las cintas, la digitalidad) los que se deterioran y deshacen; la familiaridad provista por el contacto con un entorno habitable (familiaridad propia de lo digital, sobre todo de los videojuegos con espacios tridimensionales) deviene fuente de inquietud, escenario del desmoronamiento material del texto y de su posterior clausura. No hay causa diegética o explicación para ese derrumbe, y su profundización sugiere la presencia de una forma nueva de lo siniestro donde la ambivalencia reside ya no solo en la tematización de la intimidad del hogar, sino en la posibilidad de que sea el soporte mismo el que se descomponga durante el contacto discursivo.

\section{CONCLUSIONES}

Apropiándose de un motivo como el del enrarecimiento del hogar, la producción más reciente del videojuego se aproxima por distintas vías a la generación de lo siniestro. Los casos trabajados permiten atender a formas singulares de despliegue de lo siniestro y trazan un arco que va desde su inscripción como efecto secundario, residual, pasan por una reformulación exploratoria, no narrativa, de lo fantástico, y terminan en una escena donde lo siniestro surge mayormente del deterioro de los soportes mediáticos. Si bien es posible que el videojuego haya ensayado despliegues de lo siniestro en el pasado, no fue hasta el surgimiento del llamado art game primero, y del walking simulator después, que el medio comenzó a elaborar de manera sostenida estas modalidades. De los muchos intercambios entre el campo de las artes y el videojuego, los casos analizados muestran cómo lo siniestro, una categoría estética decisiva para el siglo pasado, habilita en el presente la réplica de operaciones de otros lenguajes artísticos. Y, a su vez, así como Freud (1992) sostenía que la ficción habilitaba el surgimiento de nuevas formas de lo siniestro, del videojuego emergen hoy formas inéditas de inscripción y despliegue de lo siniestro a partir del trabajo con aspectos específicos del medio.

\section{REFERENCIAS}

Aardse, K. (2014). The Other Side of the Valley; Or, Between Freud and Videogames. Journol of Games Criticism, 1(1), 1-14. Recuperado de http://gamescriticism.org/articles/aardse-1-1

Bejarano, C. (2011). La visualidad siniestra. Revisto Figuraciones (8). Recuperado de http:// wWw.revistafiguraciones.com.ar/numeroactual/articulo.php\%3Fida\%30176\%26idn\%308\%26arch\%301 
Brown, A. y Marklund, B. (mayo de 2015). Animal Crossing: New Leaf and the Diversity of Horror in Video Games. Ponencia presentada en DiGRA 2015 Conference: Diversity of Play: Games - Cultures - Identities. Leuphana University of Lüneburg, Luneburgo, Alemania. Recuperado de http://www.digra.org/digital-library/publications/animal-crossing-new-leaf-and-the-diversity-of-horror-in-video-games

Crawford, E. (julio de 2017). Glitch Horror: BEN Drowned and the Fallibility of Technology in Game Fon Fiction. Ponencia presentada en DiGRA 2017. Digital Games Research Association, Melbourne, Australia. Recuperado de http://www.digra.org/digital-library/publications/ glitch-horror-ben-drowned-and-the-fallibility-of-technology-in-game-fan-fiction/

Cross, K. (14 de agosto de 2015). How «walking simulators» allow us to touch other worlds. Gamasutra [Entrada de blog]. Recuperado de https://www.gamasutra.com/view/ news/251191/How_walking_simulators_allow_us_to_touch_other_worlds.php

Donovan, T. (2010). Replay. The history of video games. Lewes, Gran Bretaña: Yellow Ant.

Freud, S. (1992). Obros completos de Sigmund Freud. Volumen XVII (1917-1919). Ciudad Autónoma de Buenos Aires, Argentina: Amorrortu.

Grimshaw, M. y Tinwell, A. (abril de 2009). Survival horror games. An uncanny modality. Ponencia presentada en Thinking After Dark International Conference. Universidad de Bolton, Bolton, Gran Bretaña. Recuperado de http://ubir.bolton.ac.uk/235/1/gcct_conferencepr-11.pdf

Kirkland, E. (septiembre 2009). Horror Videogames and the Unconny. Ponencia presentada en DiGRA 2009. Breaking New Ground: Innovation in Games, Play, Practice and Theory. Universidad Brunel, Londres, Gran Bretaña. Recuperado de http://www.digra.org/digital-library/ publications/horror-videogames-and-the-uncanny

Masschelein, A. (2011). The Unconcept: The Freudion Unconny in Late-Twentieth-Century Theory [El no-concepto: el siniestro freudiano en el siglo XX tardío].Nueva York, Estados Unidos: Suny Press.

Maté, D. (2017). Transformaciones de la figura del detective en el videojuego: tres casos recientes. Cuadernos del Instituto de Investigación y Experimentoción en Arte y Crítica, (1), 47-63. Recuperado de https://assets.una.edu.ar/files/file/critica-de-arte/2017/2017-cauna-cuaderno-iieac-nro-01.pdf

Maté, D. (2019). Juego, arte: tensiones en los pasajes del videojuego al museo. Lo trama de la comunicación, 23(1), 107-121. Recuperado de https://rephip.unr.edu.ar/bitstream/handle $/ 2133 / 14565 /$ Mat\%C3\%A9.pdf?sequence=3

Ravetto-Biagioli, K. (2016). The digital uncanny and ghosts effects. Screen, 57(1), 1-20.

Sloan, R. J. S. (2014). Videogames as remediated memories: Commodified nostalgia and hiperreality in Far Cry: Blood Dragon and Gone Home. Games and Culture, 10(6), 225-550.

Todorov, T. (1992). Tipología de la novela policial. En D. Link (Comp.), El juego de los coutos (pp. 35-39). Ciudad Autónoma de Buenos Aires, Argentina: La Marca.

Todorov, T. (2011). Introducción a la literatura fantástica. Ciudad Autónoma de Buenos Aires, Argentina: Paidós.

Vidler, A. (1992). The Architectural Unconny [Lo siniestro arquitectónico]. Cambridge, Inglateгга: MIT Press. 